\title{
Word-Length Distribution in Inuktitut Narratives: Empirical and Theoretical Findings
}

\author{
Peter Meyer \\ Göttingen
}

\begin{abstract}
This paper deals with the distribution of word lengeth in short native mylhulogieal and historisal Eskimo narrative texts. To my knowledge. no Eskimo-Aleut data have been the object ol quantitative linguistic mvestigation so far. Due to the strong linguistic and stylistic homogencily of the examilled texts II was assumed that these texts can be subsumed under a single law of word lengh Jistribution. il word lenglh distrihution at a text is considered as a funclion of cerliin of its properties, such as author. language. and genre. So fiar. word lenglh distribution in texts of a wide variety of languages and genres has heen demonstrated 10 follow distributions of the compound Poisson lamily of discrete prohability distributions. In view ol the morphological idiosynerasies of the Eshimo language in generil. which are responsible for an unusually high mean word length of about 4.5 lo 5.2 syllables per word in the texts, it is inlerssting to sec whether Eskimo texts show a significantly different behiviour with respect lo word length. The results demonstrate thal the Eskimo dala employed in this study tuan be filted welt by the Hyperpoissun distribution. Twn lurther discrete probability distributions will be deduced from certain morpholeggr-based assumptions aboul Eskimo. It turns oul that most of the Eskimo data tan be lïled hy these two distributions. The question to what extent lhese results point to a more grammatr-oriented lheory of word length is also distussed.
\end{abstract}

\section{THE DATA}

Al] texts have bean taken frum a collection of Eskimo Storias from Povumgnituk. Qucbec (Nungak. \& Arima. 1969: the original Eskimo spell ing of the toponym is 'Povimgnitug' I A number of ver! short narratives in the book have nol been taken into consideration. The selection of these texts for a quantitalive analysis can be jusifified on several grounds.

Firsl, all Iexts were produced by native speakers frum the town of Povirngnituy. whicl secures a cerlain linguislic homogencily of the Iexis in question. More specifically. the language of the texts can be delermined as belonging to the Nunavik group of the East (anadian Inuklitut branch of the Inuit (Inuktitut) hranch of the Eskimo (and, hence. Eskimo-Aleut) language Iamily (clí, the dialectal groupmgs intronduced in Fortescue, Jacobson. \& Kaplan. 1994 I. Apart from geographical considerations, a purely lin- guistic critcrion can be adduced to ascertan the dialectological pertinence to the Nunavik group. viz, the prohibition of two ar more subsequent closed syllahles (in other words. of saquences CCV(VICC) within the boundaries of a word. a constraint often referred to as 'Schneider's law'. For example. compare the hehaviour of the unpossessed terminalis case ending. the لdul number of which is -mmul. reducing to - $n m$ when following a closed syllable: arngnuahumnut maqrumnut I 'by Iwo big women'. Io he found in IexI I 3 in Nungak. \& Arima. 1969).

Second, all texts are tradilional anal usually well-known oral narratives of the area. myths. legends and historical accounts. The texts are eilher transcriptions of lape recordings or alphabelic Iranseriplions of lext wrillen by the slorytellers in standard Eskimo syllabic scripı. As editorial amendments in the texts were kepl to a minimum. the language of the narratives may. wilh certain restrictions, be said lo rellecl

Address correspondence to: Peler Meyer. Mittelstıabu 2, 1) 371177 Gijtingen. Germany.

Erschienen in: Journal of Quantitative Linguistics 4 (1997) 1-3, S. 143-155 
currenl oral story-telling tritditions. Thus. a certain genre-specific. stylistic uniformity of the texts is ensured, although the old story-telling skills have virtually disappeared.

The length of the selected letters is between 47 and 261 words.

\section{ANALYSIS OF THE DATA}

\section{General C'riteria}

Word length is delined here as the number of syllables in a word. In Eskimo. there are several clear-cut and completely coinciding criteriat that may serve as defining (token) words in texts. Morphologically, words are either mon-inflecting particles to be found as entries in the lexicon or morphologically analyzable entities with a (possibly zero) morph belonging to al closed class of endins: morphemes. Morphophonologically, conlacting morphemes undergo complex word-internal sandhi processes the outcomes of which are. however. not predictable on al purely phonological basis, depending instead on morpheme-specific properties. These contact processen do nol occur across word boundaries. Syntaclicully. inflected words are either nominal or verbal, which delermines their role in syntactic structures. In the standard Latin Iranscription. words are separated by spaces.'

Determining the notion of syllable is a more complex mallet. however, since the theoretical status of diphthongs (orthographically atr. aut. iav, Ua, ill, wi) and 'Iong vowels" (orthographically au, ri. $u n$ is not immediately cleas. For example, in the standard Eskimo syllahic sequences like mat are denoted by $11 \%$ symbols (mo-a): does this omply a syllable boundary beIween the I wo 'morae' Ima and a? As for the lnuit languages. not much discussion of these yuestiuns has laken place so far. Things are dilferent lor the Yup ik branch of Eskimo, where

In Eskimo. ending morphentes may be followed by

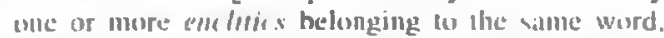
which are. III lubil alphatedie al rendering. ney senge rilled lirom the word they follow. whereas ilicy are sel ,ll hy hyplicus in slindatrd I up ik orthography. These enclilles form a vers small closed group in all Exh mono, bingunges anct are subject to wort internal sandhi any explanation of ( 'rhythmic") lenglhening and accentuation phenomena hinges crucially on a concept of syllable. Nevertheless. differen descriptive approuches are possible even in this cuse. Thus, Jacobson (I995) olfters two different ways ol describing suprasegmental phenomena in his grammar of Central Alaskan Yup ik. which, in a way. may be underslood as two diflerent views on the notion of syllable. As the decision should refer to phonological reasoning only. I shall assume here that, in the Nunavik dialect. any orthographical sequence (C) VIVIC) represents one and only one syllable: lhat is. "long vowels" and "diphthongs" are always tiul. tosyllabic (never have an internal syllable boundary). This lakes account of "Schneider"s law" mentioned above. which is thus interpreted ats a constraint on possible sequences of syllables. This happens to coincide with the notion of syllable adopled in the above-mentioned Yup ik grammar by Jicobson (1995) and is accepled. ats it seems. by the majority of Eskimo linguists. No satisfactory syllabic interprelaltion has beel found. however. for the exceedingly rare comhination of three vowel graphemes, iua. aii and unr ${ }^{2}$ I have treated them as bisyllabic. Statistically. these trigraphs accur less tham unce per texi. however.

In all calses, unly the running text without headline has been considered.

\section{Findings}

With the exception of only one text, all 33 narratives could be fitted by the I-displatied and 2 -displaced Hyperpoisson distribution. one ol the discrete probability distributions used most ollen for modelling word length. Its formula is as follows:

$$
r_{1}=\frac{11-1}{\left(n^{1 \cdot 1}, r_{1}, 11: b:(l)\right.} \cdot r=1,2 \ldots
$$

and

$$
P_{s}=\frac{u^{4-2}}{10^{1} \cdot 1,1,11: 11,611}+x=2,3 \ldots
$$

As a mallet ol lact. scejuentes of more than two volwels should not oceur in the Nunavik dialect all all. In Iwo cascs. lloe trigraphs can intleed be shown lu loe misprimts. 
The Altmann-Fitler (1994) was used with a standard chi-square test. A fit with $P\left(X^{2}\right) \geq 0.05$ wals considered as good. which is the case for 31 out of 33 texts. Fits with $0.05 \geq P\left(X^{2}\right)>0$.) () l were evaluated as still acceptable; this holds for 2 out of 33 lexts. Obviously, then, the Hyperpoisson distribution proves to be an acceptable way of modelling word length in the Eskimo text genre in question. Note, however, that in a number of texts extensive pooling of small classes was ineviable to achieve a satisfactory fit. als can be read off the respective number of degrees of freedom. For some Eskimo-internal cross-linguistic comparison. three Central Alaskan Yup ik nallrative texts taken from Jacobson (1905) have heen examined (see the last three tables below). Though the Yup ik and Inuit languages dilfer considerably in grammar and lexicon, even these control texts could be fitted by the Hyperpoisson distribution. all w ith $P\left(X^{2}\right) \geq 0.05$. Of course. lurther investigation with respect to dilferent dialects and texl genres will be necessary for a more encompassing picture of Eskimo.

The lmuktitul texts are numbered following Nungak and Arima (1969).

$$
\begin{array}{ll}
x & =\text { word length } \\
f_{1} & =\text { observed frequency } \\
N P, & = \\
& \text { the calculated frequen } \\
a \text { and } b= & \text { parameters } \\
X^{2} & =\text { chi-syuare test value } \\
D F & =\text { degrees of fieedom } \\
P & =\text { probability }
\end{array}
$$$$
\text { NP, = the calculated frequency according }
$$
to the Hyperpoisson distribution

Texi I.

\begin{tabular}{lrr}
\hline 1 & $f_{r}$ & $N f^{\prime}$ \\
\hline 2 & 18 & 18.20 \\
3 & 4.3 & 39.27 \\
+ & +7 & 49.55 \\
5 & 42 & +4.18 \\
6 & 30 & 30.40 \\
7 & 18 & 17.11 \\
8 & 11 & 8.11 \\
9 & 3 & 3.33 \\
10 & 1 & 1.79 \\
Sums & 212 & 212.000 \\
\hline
\end{tabular}

Nesce. $a=3 .\left(1.399: b=1.4\left(093: X^{2}=1.436: 1\right) F=6: P\left(X^{-1}\right.\right.$ $=0.96 .37$.
Texı 2.

\begin{tabular}{lrr}
\hline+ & $f_{1}$ & $N P_{1}$ \\
\hline 2 & 4 & 2.66 \\
3 & 7 & 6.49 \\
4 & 9 & 9.82 \\
5 & 10 & 10.76 \\
6 & 6 & 9.24 \\
7 & 12 & 6.52 \\
8 & 4 & 3.91 \\
4 & 1 & 3.60 \\
Sums & 53 & 53.00 \\
\hline
\end{tabular}

Note. $a=3.9734: b=1.6273: X^{2}=8.429: D F=5: P(X-1$ $=(1) .1341$.

\section{Texi 3}

\begin{tabular}{lrr}
\hline$x$ & $f_{1}$ & $N P_{r}$ \\
\hline 2 & 18 & 16.43 \\
3 & 20 & 23.00 \\
4 & 24 & 24.57 \\
5 & 28 & 21.23 \\
6 & 13 & 1.5 .40 \\
7 & 9 & 9.62 \\
8 & 2 & 5.28 \\
9 & 4 & 2.58 \\
10 & 2 & 1.89 \\
Sums & 120 & 120.000 \\
\hline
\end{tabular}

Nole $\left.a=4.51 .51: b=3.2253 ; X^{2}=5.949: l\right) F=\left(1: P\left(X^{2}\right)\right.$ $=0 .+289$.

Text 5.

\begin{tabular}{lrr}
\hline$r$ & $f_{1}$ & \multicolumn{1}{c}{$N{ }_{r}$} \\
\hline 2 & 7 & 8.26 \\
3 & 24 & 19.3 .3 \\
4 & 20 & 23.49 \\
5 & 20 & 19.28 \\
6 & 9 & 11.94 \\
7 & 9 & 594 \\
8 & 2 & 2.47 \\
9 & 1 & 1.29 \\
Sums & 92 & 92.001 \\
\hline
\end{tabular}

Notc. $a=2.5289 ; b=1.0813: X^{2}=4.306: D F^{*}=5: P\left(X^{2}\right)$ $=0.5062$. 
Texi 6.

\begin{tabular}{ccc}
\hline & $I_{1}$ & $N /$ \\
\hline 2 & 5 & 6.99 \\
3 & 18 & 15.28 \\
4 & 10 & 18.98 \\
5 & 16 & 16.48 \\
6 & 10 & 10.99 \\
7 & 6 & 5.95 \\
8 & 4 & 2.71 \\
11 & 1 & 1.62 \\
Sums & 79 & 79.00 \\
\hline
\end{tabular}

Nore. $a-2.8810: b=1.31901: x^{2}=1.988: D F=5 ; P\left(X^{2}\right)$ $=0.8508$.

\section{Texi 7.}

\begin{tabular}{lrr}
\hline$x$ & $f_{1}$ & $\mathrm{Nl}$ \\
\hline 2 & 7 & 6.51 \\
3 & 18 & 16.74 \\
4 & 17 & 20.98 \\
5 & 18 & 17.39 \\
6 & 15 & 10.76 \\
7 & 5 & 5.32 \\
10 & 1 & 3.30 \\
Sums81 & 81.01 & \\
\hline
\end{tabular}

Nole. $a=2.446 .3: b=0.9513: X^{2}=4.175: b F=4: P\left(X^{2}\right)$ $=0.3829$.

Texi 8 .

\begin{tabular}{lrr}
\hline$r$ & $f$ & $N P_{1}$ \\
\hline 2 & 12 & 11.12 \\
3 & 22 & 26.09 \\
4 & 38 & 31.70 \\
5 & 24 & 25.99 \\
6 & 15 & 16.08 \\
7 & 7 & 7.99 \\
8 & 5 & 3.31 \\
9 & 0 & 1.18 \\
10 & 1 & 0.54 \\
Sums & 124 & 124.00 \\
\hline
\end{tabular}

Nore $\left.a=2.521: b-1 .(1) 747: X^{2}=3.444: 1\right) F=5: P\left(X^{2}\right)=$ 0.6319 .
Texi 9.

\begin{tabular}{lcc}
\hline 1 & $f_{r}$ & N/ \\
\hline 1 & 1 & 1.07 \\
2 & 10 & 10.78 \\
3 & 22 & 21.66 \\
4 & 27 & 24.20 \\
5 & 18 & 18.71 \\
6 & 8 & 11.07 \\
7 & 7 & 5.30 \\
8 & 3 & 3.21 \\
Sums & 96 & 96.00 \\
\hline
\end{tabular}

Nope. $a=2.51 .50: b=\left(0.2515: X^{2}=1.825: 1\right) F=5: P\left(X^{2}\right)$ $=0.8728$.

Texi 10.

\begin{tabular}{lrr}
\hline$r$ & $f_{1}$ & $N{ }_{x}$ \\
\hline 2 & 20 & 17.70 \\
3 & 21 & 20.02 \\
4 & 17 & 20.20 \\
5 & 16 & 18.39 \\
6 & 15 & 15.26 \\
7 & 12 & 11.63 \\
8 & 13 & 8.19 \\
9 & 6 & 5.37 \\
10 & 4 & 7.24 \\
Sums & 124 & 124.00 \\
\hline
\end{tabular}

Nole. $a=9.3+79: h=8.26 .56: X^{2}=5.479: D F=6 ; P\left(X^{2}\right)$ $=0.48 .40$.

Texi11.

\begin{tabular}{lrr}
\hline$r$ & $f_{1}$ & $N{ }_{1}$ \\
\hline 2 & 1.3 & 11.89 \\
3 & 18 & 16.47 \\
4 & 15 & 18.31 \\
5 & 13 & 17.01 \\
6 & 21 & 13.57 \\
7 & 7 & 9.48 \\
8 & 6 & 5.90 \\
9 & 4 & 3.30 \\
10 & 1 & 1.68 \\
12 & 1 & 1.319 \\
Sums & 99 & $99.00)$ \\
\hline
\end{tabular}

Note. $\left.a=5.6470): b=4.078+: X^{2}=7 .(1) 20\right): D F=7: f^{\prime}\left(X^{2}\right)$ $=0.4268$. 
Texi 12

\begin{tabular}{lrr}
\hline$r$ & $i$, & $N P_{1}$ \\
\hline 2 & 17 & 15.33 \\
3 & 22 & 17.26 \\
4 & 1.3 & 17.85 \\
5 & 8 & 17.05 \\
6 & 16 & 15.14 \\
7 & 16 & 12.55 \\
8 & 14 & 9.76 \\
9 & 11 & 7.15 \\
11 & 4 & 4.95 \\
11 & 1 & 3.25 \\
12 & 2 & 2.112 \\
13 & 1 & 2.199 \\
Sums & 12.5 & 12.5 .00 \\
\hline
\end{tabular}

Nofe. $a=12.58(1): b=11.1698: X^{2}=15.25(): b F=9$ : $P\left(X^{2}\right)=0.08+3$.

\section{Text 13.}

\begin{tabular}{lrr}
\hline & 1, & $N /$ \\
\hline 2 & 19 & 23.81 \\
3 & 34 & 25.59 \\
4 & 15 & 24.30 \\
5 & 21 & 20.67 \\
6 & 23 & 1.5 .92 \\
7 & 9 & 11.21 \\
8 & 10 & 7.26 \\
9 & 3 & 4.36 \\
111 & 2 & 2.43 \\
11 & 1 & 1.27 \\
12 & 1 & 1.18 \\
Sums & 1.38 & 1.38 .00 \\
\hline
\end{tabular}

Nore. $a=8.1550: b=7.5865: X^{2}=12.485: b F=8$ : $P\left(X^{2}\right)=0.1 .308$.

\section{Texi 14.}

\begin{tabular}{lrr}
\hline 1 & $f$. & $N I_{1}$ \\
\hline 1 & 2 & $9 .(10)$ \\
2 & 27 & 18.192 \\
3 & 26 & 26.67 \\
4 & 34 & 31.30 \\
5 & 29 & 30.43 \\
6 & 18 & 25.26 \\
7 & 24 & 18.29 \\
8 & 1.3 & 11.74 \\
9 & 3 & 6.77 \\
10 & 5 & 3.54 \\
11 & 2 & 1.70 \\
12 & 1 & 1.28 \\
Sums & 184 & $18+.010$ \\
\hline
\end{tabular}

Norle $a=5.6711: b=2.8321: X^{2}=4.851: b F=5: P\left(X^{2}\right)$ $=0.4 .344$.
Text 15.

\begin{tabular}{lcc}
\hline & $f_{1}$ & $N P$ \\
\hline 2 & 13 & 10.88 \\
3 & 13 & 17.03 \\
4 & 19 & 17.90 \\
5 & 14 & 14.34 \\
6 & 12 & 9.18 \\
7 & 5 & 4.91 \\
8 & 1 & 2.25 \\
9 & 1 & 1.42 \\
Sums & 78 & 78.001 \\
\hline
\end{tabular}

Nore $a=3.25\left(18: h=2.0769: X^{2}=3.1(00): l\right) F=5: I^{2}\left(X^{2}\right)$ $=0.68+6$.

Texi 16.

\begin{tabular}{lcc}
\hline 8 & $f_{4}$ & $N P_{1}$ \\
\hline 2 & 1.3 & 11.78 \\
3 & 22 & 19.45 \\
4 & 20 & 23.03 \\
5 & 21 & 20.18 \\
6 & 1.3 & 14.25 \\
7 & 8 & 8.42 \\
8 & 7 & 4.28 \\
9 & 1 & 3.11 \\
Sunis & 10.5 & 105.00 \\
\hline
\end{tabular}

Norr.,$a=3.6349 ; b=2.1+79: X^{2}=4.014: D F=5 ; P\left(X^{2}\right)$ $=0.5+74$.

Text 17.

\begin{tabular}{lrr}
\hline 1 & $r$ & $N P$ \\
\hline 2 & 24 & 6.63 \\
3 & 11 & 10.54 \\
4 & 5 & 13.94 \\
5 & 10 & 15.63 \\
6 & 11 & 15.26 \\
7 & 8 & 1.3 .19 \\
8 & 17 & 10.23 \\
9 & 5 & 7.19 \\
110 & 7 & 4.62 \\
11 & 2 & 2.74 \\
12 & 3 & 2.48 \\
Sinns & 10.3 & 103.100 \\
\hline
\end{tabular}

Nofe. $a=7.5+44: b=4.7286: X^{-}=5.793: D F=1: P\left(X^{2}\right)$ $=0.01161$. 
Texı I 8

\begin{tabular}{lrr}
\hline$x$ & $f_{1}$ & $N P_{\mathrm{v}}$ \\
\hline 2 & 11 & 9.70 \\
3 & 20 & 17.65 \\
4 & 18 & 20.71 \\
5 & 15 & 17.94 \\
6 & 13 & 12.32 \\
7 & 11 & 7.00 \\
8 & 3 & 5.68 \\
Sunns & 91 & 91.00 \\
\hline
\end{tabular}

Note. $a=3.3102: b=1.8206: X^{2}=4.870: b r=4: P\left(X^{2}\right)$ $=0.3009$.

\section{Texi 19.}

\begin{tabular}{lrr}
\hline$x$ & $f_{s}$ & $N P_{\mathrm{v}}$ \\
\hline 2 & 5 & 9.22 \\
3 & 15 & 11.10 \\
4 & 7 & 10.16 \\
5 & 4 & 7.50 \\
6 & 4 & 4.63 \\
7 & 8 & 2.46 \\
8 & 4 & 1.93 \\
Sums & 47 & 47.00 \\
\hline
\end{tabular}

Noll $a=3.8107: h=3.1645 ; X^{2}=5 .(1) 26 ; D F=1 ; P\left(X^{2}\right)$ $=0 .(1250$.

\section{Texi 2I.}

\begin{tabular}{lrr}
\hline$x$ & $f_{4}$ & $N P$ \\
\hline 2 & 49 & 41.93 \\
3 & 45 & 6.3 .75 \\
4 & 73 & 62.77 \\
5 & 51 & 45.70 \\
6 & 24 & 26.39 \\
7 & 11 & 12.63 \\
8 & 7 & 5.16 \\
9 & 0 & 1.84 \\
10 & 1 & 0.83 \\
Sums & 261 & 261.00 \\
\hline
\end{tabular}

Nole. $\left.a=2.7941: b=1.8374: X^{2}=9.42 !: l\right) f=4:(3)$ $=0.0 .514$.
Texl 22.

\begin{tabular}{lrr}
\hline$x$ & $f_{1}$ & $N P_{r}$ \\
\hline 2 & 22 & 20.43 \\
3 & 23 & 24.47 \\
4 & 17 & 23.27 \\
5 & 27 & 18.36 \\
6 & 13 & 12.37 \\
7 & 4 & 7.28 \\
8 & 4 & 3.80 \\
9 & 2 & 1.78 \\
10 & 1 & 1.24 \\
Sums & 113 & 113.00
\end{tabular}

Nole, $\left.a=4.6247: b=3.8616: X^{2}=7.545: l\right) F=6: P\left(X^{2}\right)$ $=0.2733$.

\section{Texi 23.}

\begin{tabular}{lrr}
\hline .1 & $f_{8}$ & \multicolumn{1}{c}{$N J^{\prime}$} \\
\hline 2 & 12 & 8.95 \\
3 & 4 & 10.24 \\
4 & 10 & 10.05 \\
5 & 8 & 8.62 \\
6 & 12 & 6.58 \\
7 & 7 & 4.51 \\
8 & 1 & 2.81 \\
9 & 1 & 3.24 \\
Sums & 5.5 & 5.5 .00 \\
\hline
\end{tabular}

Note. $u=6.8540: h=5.9861: X^{2}=2.266: D F=1: P\left(X^{2}\right)$ $=0.1322$.

Text 25 .

\begin{tabular}{lrr}
\hline$x$ & 1, & $N P$, \\
\hline 2 & 15 & 22.93 \\
3 & 37 & 36.27 \\
4 & 51 & 41.01 \\
5 & 22 & 36.09 \\
6 & 36 & 26.00 \\
7 & 11 & 15.85 \\
8 & 10 & 8.37 \\
9 & 4 & 3.901 \\
10 & 5 & 1.63 \\
11 & 11 & 0.61 \\
12 & 0 & 0.21 \\
13 & 2 & 0.1 .3 \\
Suns & 19.3 & 193.00
\end{tabular}

Note. $a=3.9677: h=2.5086: X^{2}=3.633: b F=1: P\left(X^{2}\right)$ $=0.0567$. 
Texı 28 .

\begin{tabular}{lcc}
\hline 1 & $f_{1}$ & $N J_{1}$ \\
\hline 2 & 25 & 21.51 \\
3 & 16 & 24.73 \\
4 & 30 & 24.67 \\
5 & 21 & 21.73 \\
6 & 17 & 17.1 .3 \\
7 & 21 & 12.23 \\
8 & 4 & 7.97 \\
9 & 1 & 4.78 \\
10 & 3 & 2.65 \\
11 & 2 & 2.60 \\
Sums & 140 & 140.00 \\
\hline
\end{tabular}

Nore. $a=7.5258: b=6.5444: X^{2}=7.5(64: D) F=4: P\left(X^{2}\right)$ $=0.1089$.

Tex1 29.

\begin{tabular}{lrr}
\hline & $f_{1}$ & $N P_{1}$ \\
\hline 2 & 21 & 17.50 \\
3 & 23 & 27.19 \\
4 & 24 & 29.89 \\
5 & 20 & 25.43 \\
6 & 22 & 17.64 \\
7 & 21 & 10.3 .3 \\
8 & 5 & 5.23 \\
9 & 1 & 3.79 \\
Sums & 1.37 & 137.00 \\
\hline
\end{tabular}

Nole, $\left.a=3.7595 ; b=2.4195 ; X^{2}=7.788 ;\right) F=3 ; P\left(X^{2}\right)$ $=0.0506$.

\section{Texi 30.}

\begin{tabular}{lrr}
\hline$x$ & $f_{x}$ & $N r^{3}$ \\
\hline 2 & 12 & 16.65 \\
3 & 39 & 31.01 \\
4 & 39 & 37.70 \\
5 & 34 & 34.02 \\
6 & 19 & 24.41 \\
7 & 12 & 14.53 \\
8 & 9 & 7.39 \\
9 & 4 & 3.28 \\
10 & 3 & 2.01 \\
Sums & 171 & 171.00 \\
\hline
\end{tabular}

Nore. $a=3.5016 ; b=1.88() 1 ; X^{2}=6 .\left(1993: D Y=6 ; P\left(X^{2}\right)\right.$ $=0.4128$.
Tex131.

\begin{tabular}{lcc}
\hline 1 & $f_{x}$ & $N P_{x}$ \\
\hline 2 & 14 & 11.52 \\
3 & 12 & 15.41 \\
4 & 15 & 15.81 \\
5 & 14 & 13.16 \\
6 & 12 & 9.20 \\
7 & 4 & 5.56 \\
8 & 5 & 5.34 \\
Sums & 76 & 76.000 \\
\hline
\end{tabular}

Note. $a=4.4037: b=3.2927: X^{2}=2.685: D F=4: P\left(X^{2}\right)$ $=0.6118$.

Text 32.

\begin{tabular}{lrr}
\hline$x$ & $i_{1}$ & $N P_{1}$ \\
\hline 2 & 3.5 & 31.21 \\
3 & 36 & 37.23 \\
4 & 36 & 38.88 \\
5 & 26 & 36.11 \\
6 & 34 & 30.20 \\
7 & 32 & 22.97 \\
8 & 17 & 16.02 \\
9 & 11 & 10.31 \\
10 & 5 & 6.17 \\
11 & 2 & 3.44 \\
12 & 1 & 1.80 \\
13 & 1 & 1.66 \\
Sums & 236 & 236.001 \\
\hline
\end{tabular}

Note. $a=8.3992 ; h=7 .\left(1426 ; X^{2}=9.042 ; D F=9: P^{\prime}\left(X^{2}\right)\right.$ $=0.4288$ :

Texi 34.

\begin{tabular}{lrr}
\hline$x$ & $f_{1}$ & \multicolumn{1}{c}{$N P_{1}$} \\
\hline 2 & 13 & 2.57 \\
3 & 6 & 18.37 \\
4 & 25 & 24.14 \\
5 & 20 & 17.48 \\
6 & 10 & 8.73 \\
7 & 1 & 3.32 \\
8 & 1 & 1.39 \\
Sums & 76 & 76.00 \\
\hline
\end{tabular}

Note. $a=1.6112: b=0.2259: X^{2}=2.305: D F=2: P\left(X^{2}\right)$ $=0.3158$. 
Text 35 .

\begin{tabular}{lcc}
\hline 1 & $f_{1}$ & $N P$ \\
\hline 2 & 15 & 12.87 \\
3 & 10 & 15.92 \\
4 & 16 & 17.06 \\
5 & 18 & 10.14 \\
6 & 20 & 13.65 \\
7 & 14 & 10.45 \\
8 & 5 & 7.31 \\
9 & 1 & 4.70 \\
10 & 1 & 2.80 \\
11 & 2 & 1.55 \\
12 & 1 & 0.80 \\
13 & 0 & 0.39 \\
14 & 1 & 0.36 \\
Sums & 104 & 104.00 \\
\hline
\end{tabular}

Nole. $\quad a=8.0414 ; b=6.5\left(026: X^{2}=12.070 ; D F=8:\right.$ $P\left(X^{2}\right)=0 .|48|$.

Texi 39.

\begin{tabular}{lrr}
\hline & $f$ & $N I_{1}$ \\
\hline 2 & 6 & 5.98 \\
3 & 14 & 11.02 \\
4 & 10 & 14.98 \\
5 & 18 & 16.13 \\
6 & 12 & 14.37 \\
7 & 16 & 10.92 \\
8 & 5 & 7.23 \\
9 & 4 & 4.25 \\
10 & 2 & 2.24 \\
11 & 1 & 1.07 \\
12 & 1 & 0.81 \\
Sums & 89 & 89.00
\end{tabular}

Nole. $a=5.1729: b=2.8064: X^{2}=6.175: D F=7: P\left(X^{2}\right)$ $=(1.5194$

Texi 40.

\begin{tabular}{lrr}
\hline & $f_{1}$ & $N P_{1}$ \\
\hline 2 & 6 & 9.43 \\
3 & 8 & 12.92 \\
4 & 22 & 14.37 \\
5 & 1.3 & 13.43 \\
6 & 8 & 10.83 \\
7 & 12 & 7.68 \\
8 & 1 & 4.86 \\
9 & 6 & 2.78 \\
11 & 1 & 1.45 \\
13 & 1 & 0.69 \\
17 & 1 & 0.56 \\
Sums & 79 & 79.00
\end{tabular}

Nole: $a=5.88 .57: b=4.2957: X^{2}=7.323: D F=3: I\left(X^{2}\right)$ $=0.0623$.
Texi 41 .

\begin{tabular}{rrr}
\hline 1 & $f_{r}$ & $N P_{1}$ \\
\hline 2 & 12 & 11.80 \\
3 & 16 & 13.04 \\
4 & 16 & 1.3 .21 \\
5 & 9 & 12.34 \\
6 & 10 & 10.71 \\
7 & 8 & 8.67 \\
8 & 4 & 6.58 \\
9 & 5 & 4.69 \\
10 & 7 & 3.16 \\
11 & 2 & 4.80 \\
Sums & 89 & 89.019
\end{tabular}

Note. $a=12.1036: b=10.9510: X^{2}=9.528: D F=7$; $P\left(X^{*}\right)=0.2169$

Text 45

\begin{tabular}{rrr}
\hline 1 & 1. & $N 1$ \\
\hline 2 & 20 & 8.20 \\
3 & 4 & 12.69 \\
4 & 13 & 15.64 \\
5 & 21 & 16.03 \\
6 & 8 & 14.015 \\
7 & 9 & 10.77 \\
8 & 3 & 7.32 \\
9 & 13 & 4.48 \\
10 & 11 & 2.49 \\
11 & 3 & 2.3 .3 \\
Sums & 94 & 94.00
\end{tabular}

Nore. $:=6.0762: h=3.9297 ; X^{2}=5.694: D l=3: P^{\prime}\left(X^{2}\right)$ $=0.1275$.

Texi lor compatison: Yup' ik narrative (Jacohson, 1995, p. 4511

\begin{tabular}{ccc}
\hline$x$ & 1 & $N P$, \\
\hline 1 & 12 & 12.75 \\
7 & 58 & 51.94 \\
3 & 68 & 75.86 \\
4 & 68 & 67.50 \\
5 & 44 & 43.19 \\
6 & 24 & 21.57 \\
7 & 10 & 8.8 .3 \\
8 & 1 & 3.06 \\
10 & 1 & 1.30 \\
Sums & 286 & 286.00 \\
\hline
\end{tabular}

Nore. $a=2.2774: b=0.5593: X^{2}=3,453, b F=6: P\left(X^{2}\right)$ $=0.75013$. 
Text lor comparison: Yup ik narrative (Jacobson, 1995. p. 4.54$)$

\begin{tabular}{|c|c|c|}
\hline$x$ & $f_{1}$ & $N I^{\prime}$ \\
\hline I & 4 & $30 .(x)$ \\
\hline 2 & 102 & 60.07 \\
\hline 3 & 77 & 76.79 \\
\hline 4 & 60 & 72.09 \\
\hline 5 & 43 & 5.348 \\
\hline 6 & 33 & 32.79 \\
\hline 7 & 23 & 17.1 .3 \\
\hline 8 & + & 7.80 \\
\hline y) & 2 & 3.14 \\
\hline 11) & 1 & 1.71 \\
\hline Sums & 3.55 & $355 .(1)$ \\
\hline
\end{tabular}

Nolc. $a=3.5353 ; / l=1.76 .55: X==5.36) 2 ; l l=2 ; P\left(X^{-2}\right)$ $=0.01675$.

Texi lor comparison: Yup'ik narralive (Jacohoon. 1995. p. 4561

\begin{tabular}{lrr}
\hline$x$ & $f_{1}$ & $N P_{1}$ \\
\hline 1 & 9 & 8.71 \\
2 & 56 & 51.55 \\
3 & 77 & 76.79 \\
4 & 52 & 6.5 .43 \\
5 & 47 & 39.04 \\
6 & 22 & 17.92 \\
7 & 4 & 6.68 \\
8 & 2 & 2.88 \\
Sums & 260 & 269.000 \\
\hline
\end{tabular}

Note. $a=1.99 \mid 0 ; 1=\left(1.3360 ; X^{*}=7 .(12) ; l\right) /=5 ;\left(M X^{2}\right)$ $=0.2185$.

\section{SOME INTERPRETATION: RELATING I.INGUISTIC STRUCTUIRE AND WORD LENG;TH}

Previous altempts al explaining the adeyualcness of certain disctete probability distributions for modelling of word length relied to a large extent on considerations thal do not refer to spe cifically grammatical licalures of the languages in question (ct.. the summary on possible approaches in Wimmer, Köhler. Grotjahn, de Alsmann (1944). Thus, one may assume that. in at given language. any class of word length is propurtional to all other classes of smaller length. arriving therehy. e.g.. at generalized Poisson dis-
Iributions. However. although such an assumption bears a certain plausibility residing mosily in considerations of mathematical simplicity and previous experience with linguistic synergetics in general. it does not reflect any specifically morphological. syntactic or other properties of the languatge in question.

In what follows. some perspectives un more grammar-oriented inodels of word lenglh distribution will he outlined. In particular. I shall draw attention (o) the morphemic organisation of words. Indeed. it is just the strongly agglutinative structure of Eskimo words that suggests such a line of reasoning. In general. any Eskimo word can easily be interpreted as a chain of clear-cut morphemes.

Some brief remarks on the principles of word formation in Eskimo may he in order here. Words either belong 10 a closed class of uninllected "adverhial" particles or they are inllected. in which case they are either nouns or verbs. An inflected word consists of a (nominal or verbal) stem and a (nominal or verhal) ending (E). In the most elementary case. a stem just consists of one morpheme called (nominal or verbal) root or hase (B). symbolically: $\left.|| B\right|_{\text {sicom }}$ - El. e.g. miga $^{3}$ HU SHAND-MY:ABS 'my husband' (absolulive (asc). Usually, however. bases are suffixed hy one or more out of several hundred available posibasir morphemes, here symbolized is P. Io form successively more complex slems. Word structure might then be characterized as follow:

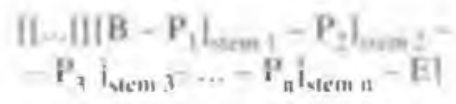

Thus, slems may be expanded through recursive right-branching. such thut lach postbase may be characterized as being the (morphological. semantic' regens of the whole preceding stem and hence the "he ad" of the slem that consists of the postbase itself and all preceding morphemes. Postbases are either nominal or verbal in that they determine whether the stem they are head of is nominal or verbal They are

\footnotetext{
" Hyplens indicante morphemoc analysis and are nor part of slandard alphabetic urtlongraphy. Examples in lliese two paragraphs are taken lirom the phonokgeically more regular Ieloolik dialect of Inuis.
} 
usually also determined as to whether the stem or naked base they are suffixed 10 must be verbal or nominal. There is no theoretical limit as to the number of admissible postbases in a word. Endings may be followed by an enclitic' suftix, such as - lu "and": uigalu and my husband".

For an example, we may take the base aiHUSBAND from above as a starling point and add a "nominal-to-verbal" postbuse -qaq-HAVE (1) get the stem ui-qaq-HUSBAND-HAVE 'to have a husband'; of. "ui-qaq-lumga HUSBAND-HAVEISG:PRES:ITR 'I have a husband' with the ending -junga. A 'verbal-to-verbal' postbase such as -juma- WANT might then be added to this stem to yield an even more complex stem uiqa-ruma- "10 want to have a husband", as in uiqarmanajungalu" I want to have a husband, $100^{\circ}$ (note the word sandhi found in these examples). This may be continued to form ai-qu-rumalaum/g/-ngit-lumga HUSBAND-HAVE-WANTPAST-NEG-ISG:PRES:ITR 'I didn't want to have a husband' - or even much larger constructions. This way, Eskimo packs much of what would be sentence structure else where into productive and legular word-internal derivational processes.

Two very simple and plausible principles of word length constitution in Eskimo are posiulated:

(1) Word length in Eskimo as expressed in terms of morpheme number (henceforth called morphemic distriburion) can be modelled by a (possibly one-displaced) simple Poisson distribution with parameter $b$.

(2) Morpheme length in Eskimo as expressed in terms of syllable number (henceforth called syllabic distribution) can be modelled by a (possibly one-displaced) simple Poisson distribution with parameter $m$.

Thus, eich word has an average of $b$ morphemes $(b+1$ in the one-displaced case), with a mean syllable number of $m(m+1$ in the one-displaced cuse) per morpheme. I assume mulual independence of all random variables here the lengll of a morpheme is influenced neither by the length of other syllables nor by the number of syllables in the word).
The advantages of this approach are obvious. First, the (wo parameters involved receive a direct linguistic interpretation and can. at least in principle (but see conclusion) be verified on the dala. Second. the asisumptions made here may be viewed as governed hy a single principle stating that the length of a certain type of linguistic unit as expressed in terms of the number of its subunits is Poisson-distributed.

I shall now give explicit forms for the word length distribution that is determined by the above postulates, starting with the more involved case of a one-displaced syllabic distribution, where zero-syllable morphemes (which are rare in standard analyses of Eskimo morphology) are disallowed. such that a word of $\boldsymbol{n}$ syllahles may consist of any number of morphemes between I and $n$.

Let us denote the probability of word length i $(l=1.2 .3, \ldots)$ with given parameters $b$ and $m$ as explained above by $P(b, m, i)$. Lel $\pi(a, r)=$ e $\frac{t}{a-1}$ denote the one-displaced simple Poisson distribution with expectancy value $a+1$. where $x=1.2$... According to the two general principles proposed above. we then have

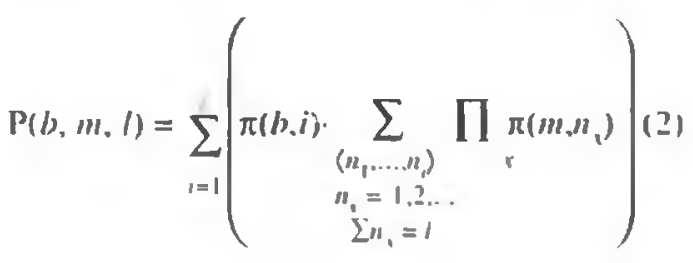

This is to be understood as follows. To find. e.g. . the probability $P(b, m, 5)$ of a word lenglh of live syllables for given values of $m$ and $h$. one sums up the prohabilities of all 'morpheme configuralions" possible for words with live syllables, multiplied by the probubility $\pi(h, i)$ of the respeclive number 1 of morphemes per word as determined by the configuration. Here a 'morpheme configuralion' is simply a sequence of morpheme lengths. such as 2-1-2. meaning 'first a two-syllable morpheme, then a monosyllabic one. linally again a bisyllabic one', which must be distinguished from.e.g., 1-2-2. The probability of such configurations is calculated by multiplying the respective morpheme length probabilities, e.g.. for 1-2-2 or 2-1-2: $\pi(m, 1) \times \pi(m, 2) \times \pi(m, 2)$. 
In (2), the calculation is split into (wo parts. We summarize over the number of possible morphemes (viz., I $10 /$ in a word of / syllables (outer sum). Within each possible number of morphemes $i$ in the word. the morphemic proh. ahility for which is $\pi(b, 1)$, the inner sum runs over all possible ordered i-fold partirions $\left\langle n_{1}, \ldots\right.$ $n_{i}$ ) of $l$. that is, over all ordered $i$-luples of pusilive, non-zero integers $"$, whose sum is /. (Thus, 1-2-2 is an ordered 3-fold partition of 5.) These partitions represent the available arrangements of 1 morphemes within a word of / syllables. The probability of each arrangement is. of course. the product of the probabilities for the compossing morphemes, which. in lurn, only depend on the length of the respective morphemes themselves as determined by the partition in ques(iion.

Though (2) gives a straightforward accoun of our distribution, it is neither easy (o) handle mathematically nor trivial to calculate. As there are $2^{\prime}$ possible partitions of an integer $l$. computing times explode exponentially tor growing $l$ in (2). As a consequence, we had rather look for some more stringent formula. To begin with. the inner sum in (2). henceforth abbreviated as $p(m, 1, i)$, can be interpreted as a probability distribution with parameters $m$ and $i$. evaluated for value I. This distribution obviously is the sum of $i$ mutually independent onc-displaced Poisson distributed random variables with pirameter $m$. Since the prohahility gentraning lunctions (pgf) of mutually independent distributions multiply when their random variathles are

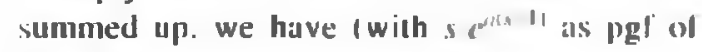
$\pi(a . x))$ :

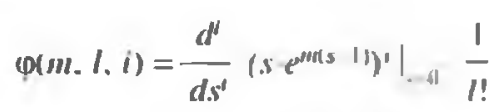

In order to Iurther simplily (3). we note that $\frac{d}{d s^{\prime}}$ $(/(s) \cdot g(s))=\sum_{x=0}\left(\begin{array}{l}1 \\ x\end{array}\right) \cdot f^{(1)}(s) \cdot g^{(1-r)}(s)$ for arbitarily often diflerentiable Iunctions / and $g$ and that $\frac{d^{\prime}}{d s^{\prime}} s^{\prime}$ will he 1 ! for $i=l$ and simply o for all olher I. We thus oblain

4 Here. $f(1)(s)$ is. of course, the usual ahbreviation ol $\frac{d}{d x^{n}} f(s)$. ind nol a descending faclorial

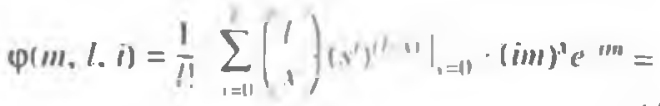

$$
\begin{aligned}
& =\frac{1}{1 !}\left(\begin{array}{c}
1 \\
1-i
\end{array}\right)(1+i m)^{2}+e^{-}
\end{aligned}
$$

Replacing the inner sum (plm. I. I) In (1) with our last formula in (4), we finally have

$$
\begin{aligned}
& \mathrm{P}(h, m, l)=\sum_{i=1}^{l}(\pi(h, l)-\varphi(m, l, i))= \\
& \sum_{i}^{1} \frac{e^{\prime \prime} b^{1}}{(1-1) !}, \frac{1}{1 !} \frac{1 !}{i !(1-1) !} i ! \cdot(i m)^{\prime} \cdot e^{\prime m}
\end{aligned}
$$

After some lidying up our result is a surprisingly simple formula with calculation limes growing in a linear fashion with growing /, viz.

$$
\begin{aligned}
& P(b, m, l)=\frac{e^{n}}{1 !} \sum_{i=1}^{i}\left(\begin{array}{l}
1 \\
i
\end{array}\right)\left(\cdot b ^ { \prime } \left((m m)^{n}+e^{n+m} .\right.\right. \\
& l=1,2,3 \ldots
\end{aligned}
$$

The case of a word length distribution with nondisplaced syllabic distribution. here denoted as $P$ (b. m. l), is much easier to cope with. I.inguistically speaking. $P$ may be interpreted as allowing for zero-fength morphemes. Thus. il al word has Iwo syllables, there is, according I0 $P^{*}$. a certain. albeit small probabilıy that the word is composed of, say. I.37 morphemes, 1.36 of which do not contain a syllable core. In general, any uord is allowed to consist of an arbiIrarily high number of morphomes. Inslead of (2). we slant from the following:

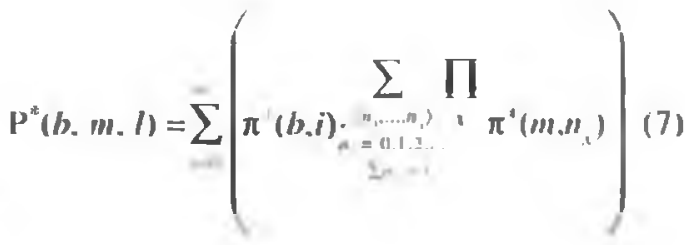

Here, $\pi(a, r)$ denoles the Poisson distribution. $\pi(a, . r)=e^{-a b} \frac{w}{i t}$. For the sake of simplicity, I also assume non-displaced morphemic distribution here. The inner sum in (7), henceforth abhrevialed as $\varphi(m, l, 1)$, may. accordingly, he expressed as 


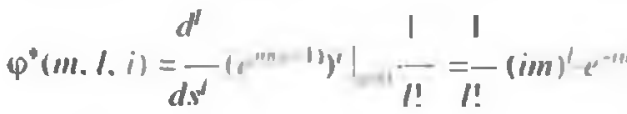

Inserted into (7), this immediately yields

$$
P(b, m, l)=\sum_{i=11}^{\infty} \frac{e^{-b} b^{\prime}}{i !} \frac{I}{1 !} \cdot i^{\prime} m^{\prime}\left(e^{-m}\right)^{\prime}
$$

After a little bit of reordering. the final formula is

$$
\begin{aligned}
& P^{\prime}(h, m, l)=\frac{e^{-h}-1 m^{\prime}}{l !} \sum_{i=1} \frac{l^{l}\left(h e^{-m}\right)}{i !} . \\
& I=0.1 .2 \ldots
\end{aligned}
$$

However. this is just the formula of the wellknown Neyman distribution type A with two parameters. the use of which for modelling word length has lluus been motivated partially on linguistic grounds. Note. however. that $P(b . m, /)$ in $(10)$ is nol simply some shifting or reparametrizalion variant of $P^{\prime \prime}(b, m, l)$.

It is clearly of paramount importance to in vestigate the empirical significance of the two proposed distributions, $P(b, m, l)$ and $P(b, m$. 1). A preliminary examination has shown that

- oul of 20 Eskimo texis checked. at least 18 may be fitted by $P(b, m$. I). out of these at least 1.3 well $\left(P\left(X^{2}\right) \geq 0.05\right):^{01}$

- out of 20) Eskimo texis counted. at leatst 18 may be fitted to $P^{\prime \prime}(b, m$. (): out of these at least 1.3 well $\left(P\left(X^{2}\right) \geq 0.05\right):^{7}$

- for a large range of possible choices for the parameters $m$ and $b . P(b, m, l)$ is a good approximation to the Hyperpoisson distribution;

- for a large range of possible choices for the parameters $m$ and $b . P(h, m$. l) is a good approximation to the Hyperpoisson distu ibulion.

- The anc-displaced variant al the distribution has been employed throughout, with the exeeption of $\mid \mathrm{exl}$ If. the only checked text with a class of monosyllahic words. Resules for Ihis disiribution were had only where Hyperpoisson lit was also problematic. al leasi belore pooling clissies.

h Except for țxi It. The Iwo-displaced variant of this distribution was used. Resulss for this distributisn were bad also only where Hyperpoisson fil wis also prohlematic. at leas belore pouling clasices.
What is the moral to be drawn from these data! First, $P(h, m, l)$ and $P(b, m, I)$ can indeed. 10 some degree of satistacion. be regarded as malhematical models of word length in the East Calnadian Inuktitut narrative texts under investigalion. The small amount of lexts examined does not suffice lo compare these distributions stalistically - they seem to do their jub equally well. Second. the dala hill all an unusual. but perhaps interesling. way of explaining fit of cerlaill other distributions: Distributions such as the Hyperpoisson one may be "explained" by demonstralling that they approximate and are approximated well by another. well-motivated distribution. Third, the above mathematical teasoming als represented by (2) and (7) defines. if looscly. a family of probubilily distributions that seents to be al good starting point for word length modelling in non-isolating languages. This family is not the Neyman family of distributions, since $P(b, m$. / as given in (6) does not belong there. This might be un indication as 10 which of the several possible motinations or interpretarions for the Neyman A distribution should he preferred in the Eskimo case: Il" several different distributions belonging 10 a lamily $f$ turn out 10 be acceptable models for the phenomenon to be accounted for. then acceptable motivations for each of these distributions should be extendable to $F$ as a whole.

\section{CONCLUSION}

The Hyperpoissun distribution turns out to be a remarkably good model for the Eskimo texi genre examined here. By way of theorelical reasoning. two further distribulions that reflect the morphemic structure of Eskimo words have been proposed. These distributions. 100, have proven to be acceptable models of word length in the Eskimo texts. Furıher empirical investigation will be necessary to determine whether these or related distributions may he used lo model word length in other agglutimative languages.

It should be stressed. however, that the models proposed still do not present anything similar to an explanation of the regularities of word length in Eskimo. First. many other possible 
parameters (attractors) have simply heen lefi out of consideration, such as the intricucies of word formattion in Eskimo hinted al in the section on dilla analysis; the syntaclic and phonological subsyslems of the language: and many more. Second. no further justification has heen adduced for choosing the simple Poisson distribution. instead of, e.g. . the Borel distribution. for both the syllabic and the morphemic distribulion. Deeper justifications lor this choice will probably have to be founded on general synergetic process modelling.

It might seem that a good way to check the validity of these models is to make sure that the interpretation of their respective parameters is empirically valid. As for $P(b, 1,1 /$, a preliminary fitting of the data gives parameters thall indecd sound realistic (between 1.16 and 3.78 for $b+1$. that is the mean number of morphemes per word: between $1 .(09$ and 3.36 for $m+1$, the mean number of syllables per morpheme). However, the usual linguistic analyses of word structure in Eskimo are not very likely to presuppose a notion of morpheme which would be relevant for a stochastic investigation since the role that the morpheme concept has (o) plity in the (wo) different frameworks of" "iraditional" and synergetic linguistics are not identital. To mention a Irivial example: If the presence of a certain "Iratditional" morph $M_{1}$ conditions a very high prob- ahility for it to he followed hy another morph $M$, in the very same word. then the sequence $M_{1} M$, might constitute a single morph from the point of view of a stochastic approach. As at consequence. the very notion of validating or jusrifving a synergetic or, more general. stochastic model for a 'real- 1 orld' linguistic phenomenon will remain highly theory-dependent and theory-laden. Therefore, probably no simple method of relaning probabilistic models of language to 'traditional' linguistic descriptions will await us in the near future.

\section{REFERENCES}

Fortescue, M.. Jacobson. S.A.. \& Kaplan. L. 1 1904) ( pararive Eskinns dictiomary Wilh Alewr conpuates. Fuirhanks

Jacobson. S.A. (1995). A practical grammar of the (e")iral Alaskun rup'ik Exkinno language (n ilh Yup'ik readings uriten by Anna W Jacobson!. Fairbanks.

Nungak. Z.. \& Arimi. E. (1969) Unikkutuat somen enumgmk unvingualii Purirngninurngmit. Eskimo suries from Pon'ungniruk. Quebec: illustrated in soapstone carvings. Ouawa.

Wimmer. G.. Köhler. R.. Grotjahn. R. Alımann. G. (1904). Towards a theory of werd lengh disirihution. Jourmal of Qumbirurive Linguistics. I. 98-106

Soltwale:

Altmian-Filler: 1994. Liidenscheid. 